\title{
Development of metabolically engineered Corynebacterium glutamicum for enhanced production of cadaverine and its use for the synthesis of bio- polyamide 510
}

Hee Taek Kim ${ }^{1,8}$, Kei-Anne Baritugo ${ }^{2,8}$, Sung Min Hyun ${ }^{1,3,8}$, Tae Uk Khang ${ }^{1,4}$, Yu Jung Sohn², Kyoung Hee Kang${ }^{1}$, Seo Young $\mathrm{Jo}^{2}$, Bong Keun Song${ }^{1}$, Kyungmoon Park ${ }^{3}$, Il-Kwon Kim ${ }^{5}$, Yong Taek Hwang 6 , Sang Yup Lee ${ }^{7}$, Si Jae Park ${ }^{2, *}$, Jeong Chan Joo ${ }^{1, *}$

${ }^{1}$ Center for Bio-based Chemistry, Division of Convergence Chemistry, Korea Research Institute of Chemical Technology, P.O. Box 107, 141 Gajeong-ro, Yuseong-gu, Daejeon 34114, Republic of Korea

${ }^{2}$ Division of Chemical Engineering and Materials Science, Ewha Womans University, 52 Ewhayeodae-gil, Seodaemun-gu, Seoul 03760, Republic of Korea

${ }^{3}$ Department of Biological and Chemical Engineering, Hongik University, 2639 Sejong-ro, Jochiwon-eup, Sejong-si 30016, Republic of Korea

${ }^{4}$ Department of Chemical and Biological Engineering, Korea University, 145 Anam-ro, Seongbuk-gu, Seoul 02841, Republic of Korea

${ }^{5}$ Bioprocess R\&D Center, DAESANG Corp., Icheon-si, Gyeonggi-do, 17384, Republic of Korea

${ }^{6}$ Lotte Chemical, 115 Gajeongbuk-ro, Yuseong-gu, Daejeon, 34110, Republic of Korea

${ }^{7}$ Metabolic and Biomolecular Engineering National Research Laboratory, SMESH CrossGeneration Collaborative Laboratory, Department of Chemical and Biomolecular Engineering (BK21 Plus Program), Institute for the BioCentury, Korea Advanced Institute of Science and Technology (KAIST), Daejeon 34141, Republic of Korea 
${ }^{8}$ Hee Taek Kim, Kei-Anne Baritugo and Sung Min Hyun equally contributed to this work.

*Correspondence should be addressed to Si Jae Park (parksj93@ewha.ac.kr) and Jeong Chan Joo (jcjoo@krict.re.kr)

4 Pages, 2 Tables are included. 


\begin{tabular}{|c|c|c|}
\hline Primer & Primer Sequence (5' to $\left.3^{\prime}\right)$ & $\begin{array}{l}\text { Target gene } \\
\text { (UniProt \#) }\end{array}$ \\
\hline 1 & 5'-CTGCAG GTGGATTTTCGCCGCTG & $5^{\prime}$ region of $l y s E$ \\
\hline 2 & 3'-GGATCC GACCTGTAATGAAGATTTCCAT & (P94633) \\
\hline 3 & 5'-GAATTC TAGCTTCACGGGTTACCGC & $3^{\prime}$ region of $l y s E$ \\
\hline 4 & 3'-CTGCAG ACTAAAGGGAACAAAAGCTG & (P94633) \\
\hline 5 & 5'-GGATCC ATGAACATCATTGCCATTATGG & $l d c C_{E c}$ \\
\hline 6 & 3'-GCGGCCGC TTATCCCGCCATTTTTAGGA & (P52095) \\
\hline 7 & 5'-GGATCC ATGAACGTTATTGCAATATT & $\operatorname{cad} A_{E c}$ \\
\hline 8 & 3'-GCGGCCGC TTATTTTTTGCTTTCTTCTT & (P0A9H3) \\
\hline 9 & 5'-GGATCC ATGAAATTATTGAGTATCGCAGCT & $o d c_{L s}$ \\
\hline 10 & 3'-GCGGCCGC CTATTTCTTGTCTAAAACTGATTCGT & (A0A0R1SYE8) \\
\hline 11 & 5'-GGATCC ATGAAATTATTGAGTATCGCAGCT & $l d c_{L s}$ \\
\hline 12 & 3'-GCGGCCGC CTATTTCTTGTCTAAAACTGATTCGT & (A0A0R1SYD4) \\
\hline 13 & 5'-GGATCC ATGCGCTCGCACCTGCTC & $l d c_{S c}$ \\
\hline 14 & 3'-GCGGCCGC TCAGGAAGCGCGGTCAAG & (Q9L072) \\
\hline 15 & 5'-GGATCC ATGAAAAACTTCCGTTTAAGCGA & $l d c_{S r}$ \\
\hline 16 & 3'-GCGGCCGC TTAAACTGCTGCTTTTTTTTTCACA & (I0GRF9) \\
\hline 17 & 5'-GGATCC ATGAATATTATTGCCATCTT & $\operatorname{cad} A_{H a}$ \\
\hline 18 & 3'-GCGGCCGC TTACTTCTGGTCTTTGATAA & (A0A097R5J6) \\
\hline 19 & 5'-GGATCC ATGAATATCATTGCCATCATGA & $l d c C_{H a}$ \\
\hline 20 & 3'-GCGGCCGC TTATGACTTCTTCGCCGCTG & (A0A097QY94) \\
\hline 21 & 5'-GGATCC ATGGCACACAGTCAGAGCAT & $l d c_{V v}$ \\
\hline 22 & 3'-GCGGCCGC TTAACCTATCATACGCTCGGACG & (A0A087IJ86 ) \\
\hline
\end{tabular}


Table S2. Properties of enzymes used in this study.

\begin{tabular}{|c|c|c|c|c|c|c|}
\hline Enzyme & $\begin{array}{l}K_{c a t} \\
(1 / \mathbf{s})\end{array}$ & $\begin{array}{c}K_{m} \\
(\mathbf{m M})\end{array}$ & $V_{\max }$ & $\begin{array}{c}\text { Optimum } \\
\text { pH }\end{array}$ & $\begin{array}{c}\text { Optimum } \\
\text { Temperature }\end{array}$ & Reference \\
\hline $\operatorname{LdcC}_{\mathrm{Ec}}$ & $\begin{array}{l}\text { No } \\
\text { data }\end{array}$ & 0.84 & 27.2 & 7.6 & 52 & Lemonier et al., 1998 \\
\hline $\operatorname{CadA}_{\mathrm{Ec}}$ & 30 & 0.42 & $\begin{array}{l}\text { No } \\
\text { data }\end{array}$ & 5.6 & 52 & Lemonier et al., 1998 \\
\hline Odc $_{\text {Ls }}$ & 0.25 & 10.3 & $\begin{array}{l}\text { No } \\
\text { data }\end{array}$ & 5.9 & 50 & Romano et al., 2013 \\
\hline $\mathbf{L d c}_{\mathrm{Ls}}$ & 1.68 & 12.7 & $\begin{array}{l}\text { No } \\
\text { data }\end{array}$ & 5.2 & 37 & Romano et al., 2013 \\
\hline $\mathbf{L d c}_{\mathrm{sc}}$ & 0.025 & 0.058 & $\begin{array}{l}\text { No } \\
\text { data }\end{array}$ & 7.5 & 30 & Burell et al., 2012 \\
\hline $\mathbf{L d c}_{\mathrm{Sr}}$ & 10.6 & 0.63 & 14.7 & 6.0 & 45 & Takasuka et al., 1999, 2004 \\
\hline LdcC $_{\mathrm{Ha}}$ & 175 & 1.7 & 24.7 & 5.8 & 37 & Wang et al., 2015 \\
\hline $\mathbf{L d c}_{V}$ & 4 & 5.8 & $\begin{array}{l}\text { No } \\
\text { data }\end{array}$ & 9.0 & 30 & Jeong et al., 2016 \\
\hline
\end{tabular}

